\title{
A DESCRIPTION OF $n$-ARY SEMIGROUPS POLYNOMIAL-DERIVED FROM INTEGRAL DOMAINS
}

\author{
JEAN-LUC MARICHAL AND PIERRE MATHONET
}

\begin{abstract}
We provide a complete classification of the $n$-ary semigroup structures defined by polynomial functions over infinite commutative integral domains with identity, thus generalizing Głazek and Gleichgewicht's classification of the corresponding ternary semigroups.
\end{abstract}

\section{INTRODUCTION}

Let $R$ be an infinite commutative integral domain with identity and let $n \geqslant 2$ be an integer. In this note we provide a complete description of all the $n$-ary semigroup structures defined by polynomial functions over $R$ (i.e., the $n$-ary semigroup structures polynomial-derived from $R$ ).

For any integer $k \geqslant 1$, let $[k]=\{1, \ldots, k\}$. Recall that a function $f: R^{n} \rightarrow R$ is said to be associative if it solves the following system of $n-1$ functional equations:

$$
\begin{aligned}
& f\left(x_{1} \ldots, f\left(x_{i}, \ldots, x_{i+n-1}\right), \ldots, x_{2 n-1}\right) \\
& \quad=f\left(x_{1} \ldots, f\left(x_{i+1}, \ldots, x_{i+n}\right), \ldots, x_{2 n-1}\right), \quad i \in[n-1] .
\end{aligned}
$$

In this case, the pair $(R, f)$ is called an $n$-ary semigroup.

The introduction of $n$-ary semigroups goes back to Dörnte 1 and led to the generalization of groups to $n$-ary groups (polyadic groups). The next extensive study on polyadic groups was due to Post [10] and was followed by several contributions towards the description of $n$-ary groups and similar structures. To mention a few, see $2,6,8,9,11$.

We now state our main result, which provides a description of the possible associative polynomial functions from $R^{n}$ to $R$. Let $\operatorname{Frac}(R)$ denote the fraction field of $R$ and let $\mathbf{x}=\left(x_{1}, \ldots, x_{n}\right)$.

Main Theorem. A polynomial function $p: R^{n} \rightarrow R$ is associative if and only if it is one of the following functions:

(i) $p(\mathbf{x})=c$, where $c \in R$,

(ii) $p(\mathbf{x})=x_{1}$,

(iii) $p(\mathbf{x})=x_{n}$,

(iv) $p(\mathbf{x})=c+\sum_{i=1}^{n} x_{i}$, where $c \in R$,

(v) $p(\mathbf{x})=\sum_{i=1}^{n} \omega^{i-1} x_{i}$ (if $n \geqslant 3$ ), where $\omega \in R \backslash\{1\}$ satisfies $\omega^{n-1}=1$,

(vi) $p(\mathbf{x})=-b+a \prod_{i=1}^{n}\left(x_{i}+b\right)$, where $a \in R \backslash\{0\}$ and $b \in \operatorname{Frac}(R)$ satisfy $a b^{n}-b \in R$ and $a b^{k} \in R$ for every $k \in[n-1]$.

Date: January 27, 2011.

2010 Mathematics Subject Classification. 20N15.

Key words and phrases. $n$-ary semigroup, $n$-ary group, associativity, polynomial function.

Communicated by Mikhail Volkov.

Corresponding author: Jean-Luc Marichal (Phone: +352 466644-6662). 
The Main Theorem shows that the associative polynomial functions of degree greater than 1 are symmetric, i.e., invariant under any permutation of the variables.

Example 1. The third-degree polynomial function $p: \mathbb{Z}^{3} \rightarrow \mathbb{Z}$ defined on the ring $\mathbb{Z}$ of integers by

$$
p\left(x_{1}, x_{2}, x_{3}\right)=9 x_{1} x_{2} x_{3}+3\left(x_{1} x_{2}+x_{2} x_{3}+x_{3} x_{1}\right)+x_{1}+x_{2}+x_{3}
$$

is associative since it is the restriction to $\mathbb{Z}$ of the associative polynomial function $q: \mathbb{Q}^{3} \rightarrow \mathbb{Q}$ defined on the field $\mathbb{Q}$ of rationals by

$$
q\left(x_{1}, x_{2}, x_{3}\right)=-\frac{1}{3}+9 \prod_{i=1}^{3}\left(x_{i}+\frac{1}{3}\right) .
$$

The classification given in the Main Theorem was already obtained for ternary semigroups (i.e., when $n=3$ ) by Głazek and Gleichgewicht [7. Surprisingly, the classification for arbitrary $n$ remains essentially the same except for certain solutions of type $(v)$ (already mentioned in [1]), whose existence is subordinate to that of nontrivial roots of unity. Note that, when $n$ is odd, $(v)$ always provides the solution

$$
p(\mathbf{x})=\sum_{i=1}^{n}(-1)^{i-1} x_{i},
$$

which was the unique solution of type $(v)$ found in [7 for $n=3$.

In Section 2 we give the proof of the Main Theorem. In Section 3 we analyze some properties of these $n$-ary semigroup structures: we show that they are medial, determine the $n$-ary groups defined by polynomial functions, and discuss irreducibility issues for these $n$-ary semigroups.

\section{Technichlities And Proof of the Main Theorem}

Throughout this section, with every function $f: R^{n} \rightarrow R$ we associate $n$ functions $f_{i}: R^{2 n-1} \rightarrow R, i \in[n]$, defined by

$$
f_{i}\left(x_{1}, \ldots, x_{2 n-1}\right)=f\left(x_{1} \ldots, f\left(x_{i}, \ldots, x_{i+n-1}\right), \ldots, x_{2 n-1}\right) .
$$

It follows that $f$ is associative if and only if $f_{1}=\cdots=f_{n}$.

It is clear that the definition of $R$ enables us to identify the ring $R\left[x_{1}, \ldots, x_{n}\right]$ of polynomials of $n$ indeterminates over $R$ with the ring of polynomial functions of $n$ variables from $R^{n}$ to $R$. Recall that, for any integer $d \geqslant 0$, a polynomial function $p: R^{n} \rightarrow R$ of degree $\leqslant d$ can be written as

$$
p(\mathbf{x})=\sum_{j_{1}+\cdots+j_{n} \leqslant d} c_{j_{1}, \ldots, j_{n}} x_{1}^{j_{1}} \cdots x_{n}^{j_{n}}, \quad c_{j_{1}, \ldots, j_{n}} \in R .
$$

For every $i \in[n]$, we denote the degree of $p$ in $x_{i}$ by $\operatorname{deg}\left(p, x_{i}\right)$. We also denote the degree of $p$ by $\operatorname{deg}(p)$.

Proposition 2. For every associative polynomial function $p: R^{n} \rightarrow R$, we have $\operatorname{deg}\left(p, x_{i}\right) \leqslant 1$ for every $i \in[n]$. Moreover, if $\operatorname{deg}\left(p, x_{n}\right)=0\left(\right.$ resp. $\left.\operatorname{deg}\left(p, x_{1}\right)=0\right)$, then $p$ is either a constant or the projection on the first (resp. the last) coordinate.

Proof. Let $p: R^{n} \rightarrow R$ be an associative polynomial function and let $p_{1}, \ldots, p_{n}$ be the polynomial functions associated with $p$ as defined in (2). For every $i \in[n]$, we let $d_{i}=\operatorname{deg}\left(p, x_{i}\right)$. By associativity, we have

$$
\begin{array}{ll}
d_{1}=\operatorname{deg}\left(p_{i}, x_{1}\right)=\operatorname{deg}\left(p_{1}, x_{1}\right)=d_{1}^{2}, & i \in[n] \backslash\{1\}, \\
d_{n}=\operatorname{deg}\left(p_{i}, x_{2 n-1}\right)=\operatorname{deg}\left(p_{n}, x_{2 n-1}\right)=d_{n}^{2}, & i \in[n] \backslash\{n\},
\end{array}
$$


which shows that $d_{1} \leqslant 1$ and $d_{n} \leqslant 1$.

Again by associativity, we have

$$
\begin{array}{ll}
d_{i} d_{n-i+1}=\operatorname{deg}\left(p_{i}, x_{n}\right)=\operatorname{deg}\left(p_{1}, x_{n}\right)=d_{1} d_{n}, & i \in[n], \\
d_{i}=\operatorname{deg}\left(p_{i+1}, x_{i}\right)=\operatorname{deg}\left(p_{i}, x_{i}\right)=d_{1} d_{i}, & i \in[n-1], \\
d_{i}=\operatorname{deg}\left(p_{i-1}, x_{n+i-1}\right)=\operatorname{deg}\left(p_{i}, x_{n+i-1}\right)=d_{i} d_{n}, & i \in[n] \backslash\{1\} .
\end{array}
$$

If $d_{1}=d_{n}=1$, then the first set of equations shows that $d_{i}=1$ for every $i \in[n]$. If $d_{n}=0$, then the third set of equations shows that $p$ is of the form $p(\mathbf{x})=c_{1} x_{1}+c_{0}$ and hence we can conclude immediately. We proceed similarly if $d_{1}=0$.

By Proposition 2 an associative polynomial function $p: R^{n} \rightarrow R$ can always be written in the form

$$
p(\mathbf{x})=\sum_{j_{1}, \ldots, j_{n} \in\{0,1\}} c_{j_{1}, \ldots, j_{n}} x_{1}^{j_{1}} \cdots x_{n}^{j_{n}}, \quad c_{j_{1}, \ldots, j_{n}} \in R .
$$

Using subsets of $[n]$ instead of Boolean indexes, we obtain

$$
p(\mathbf{x})=\sum_{J \subseteq[n]} c_{J} \prod_{j \in J} x_{j}, \quad c_{J} \in R .
$$

In order to prove the Main Theorem, we only need to describe the class of associative polynomial functions of the form (3).

To avoid cumbersome notation, for every subset $S=\left\{j_{1}, \ldots, j_{k}\right\}$ of integers and every integer $m$, we set $S+m=\left\{j_{1}+m, \ldots, j_{k}+m\right\}$. Also, for every $i \in[n]$, we let

$$
\begin{aligned}
& A_{i}=\{1, \ldots, i-1\}=[i-1], \\
& B_{i}=\{i, \ldots, i+n-1\}=[n]+i-1, \\
& C_{i}=\{i+n, \ldots, 2 n-1\}=[n-i]+n+i-1,
\end{aligned}
$$

with the convention that $A_{1}=C_{n}=\varnothing$.

Lemma 3. If $p: R^{n} \rightarrow R$ is of the form (3), then for every $i \in[n]$ the associated function $p_{i}: R^{2 n-1} \rightarrow R$ is of the form

$$
p_{i}\left(x_{1}, \ldots, x_{2 n-1}\right)=\sum_{S \subseteq[2 n-1]} r_{S}^{i} \prod_{j \in S} x_{j}
$$

and its coefficients are given in terms of those of $p$ by

$$
r_{S}^{i}= \begin{cases}c_{J_{S}^{i} \cup\{i\}} c_{K_{S}^{i}}, & \text { if } S \cap B_{i} \neq \varnothing, \\ c_{J_{S}^{i} \cup\{i\}} c_{\varnothing}+c_{J_{S}^{i}}, & \text { otherwise, }\end{cases}
$$

where $J_{S}^{i}=\left(S \cap A_{i}\right) \cup\left(\left(S \cap C_{i}\right)-n+1\right)$ and $K_{S}^{i}=\left(S \cap B_{i}\right)-i+1$.

Proof. We first note that

$$
p\left(x_{i}, \ldots, x_{n+i-1}\right)=\sum_{K \subseteq[n]} c_{K} \prod_{k \in K} x_{k+i-1}=\sum_{K \subseteq[n]} c_{K} \prod_{k \in K+i-1} x_{k} .
$$

Then, partitioning $J \subseteq[n]$ into $J \cap A_{i}, J \cap\{i\}$, and $J \cap\left(C_{i}-n+1\right)$, we obtain

$$
\begin{aligned}
p_{i}\left(x_{1}, \ldots, x_{2 n-1}\right)= & \sum_{J \subseteq[n]} c_{J} \prod_{j \in J \cap A_{i}} x_{j} \prod_{j \in(J+n-1) \cap C_{i}} x_{j} \prod_{j \in J \cap\{i\}}\left(\sum_{K \subseteq[n]} c_{K} \prod_{k \in K+i-1} x_{k}\right) \\
= & \sum_{J \subseteq[n], J \ni i} \sum_{K \subseteq[n]} c_{J} c_{K} \prod_{j \in J \cap A_{i}} x_{j} \prod_{j \in(J+n-1) \cap C_{i}} x_{j} \prod_{k \in K+i-1} x_{k} \\
& +\sum_{J \subseteq[n], J \ngtr i} c_{J} \prod_{j \in J \cap A_{i}} x_{j} \prod_{j \in(J+n-1) \cap C_{i}} x_{j} .
\end{aligned}
$$


The result is then obtained by reading the coefficient of $\prod_{i \in S} x_{i}$ in the latter expression.

Proposition 4. Let $p: R^{n} \rightarrow R$ be an associative polynomial function of the form (3). If $c_{[n]}=0$, then $\operatorname{deg}(p) \leqslant 1$.

Proof. We assume that $c_{[n]}=0$ and prove by induction that $c_{J}=0$ for every $J \subseteq[n]$ such that $|J| \geqslant 2$. Suppose that $c_{J}=0$ for every $J \subseteq[n]$ such that $|J| \geqslant k$ for some $k \geqslant 3$. Fix $J_{0} \subseteq[n]$ such that $\left|J_{0}\right|=k-1$. We only need to show that $c_{J_{0}}=0$.

Assume first that $\ell=\min \left(J_{0}\right) \leqslant(n+1) / 2$.

(i) Case $\ell=1$. Let

$$
S=J_{0} \cup\left(\left(J_{0}+n-1\right) \backslash\{n\}\right) \subseteq[2 n-1] .
$$

We have $S \cap A_{1}=\varnothing, S \cap B_{1}=J_{0}$, and $\left(S \cap C_{1}\right)-n+1=J_{0} \backslash\{1\}$. By Lemma 3. we have $r_{S}^{1}=c_{J_{0}}^{2}$. Setting $m=\min \left([n] \backslash J_{0}\right)[1]$ we also have $\left|S \cap A_{m}\right|=\left|A_{m}\right|=m-1$ and $\left|\left(S \cap C_{m}\right)-n+1\right|=\left|J_{0}\right|-(m-1)$. Moreover, $S \cap B_{m} \neq \varnothing$ for otherwise we would have $J_{0}=\{1\}$, which contradicts $\left|J_{0}\right| \geqslant 2$. Thus, using Lemma 3, associativity, and the induction hypothesis, we have $r_{S}^{m}=0$ and therefore

$$
c_{J_{0}}^{2}=r_{S}^{1}=r_{S}^{m}=0 .
$$

(ii) Case $1<\ell \leqslant(n+1) / 2$. Let

$$
S=\left(J_{0}+\ell-1\right) \cup\left(\left(J_{0}+n-1\right) \backslash\{n+\ell-1\}\right) \subseteq[2 n-1] .
$$

We proceed as above to obtain $r_{S}^{\ell}=c_{J_{0}}^{2}$. By associativity it is then sufficient to show that $r_{S}^{2 \ell-1}=0$. Using the notation of Lemma 3, we can readily see that $\left|K_{S}^{2 \ell-1}\right| \geqslant\left|J_{0}\right|$. Hence by Lemma 3 we only need to show that $c_{K_{S}^{2 \ell-1}}=0$. If $\left|K_{S}^{2 \ell-1}\right|>\left|J_{0}\right|$, then we conclude by using the induction hypothesis. If $\left|K_{S}^{2 \ell-1}\right|=\left|J_{0}\right|$, then we can apply case $(i)$ since $\min \left(K_{S}^{2 \ell-1}\right)=1$.

If $\ell>(n+1) / 2$, we proceed symmetrically by setting $\ell^{\prime}=\max (J)$ and considering the cases $\ell^{\prime}=n$ and $(n+1) / 2 \leqslant \ell^{\prime}<n$ separately.

Proposition 5. A polynomial function $p: R^{n} \rightarrow R$ of the form (3) with $c_{[n]}=0$ is associative if and only if it is one of the functions of types $(i)-(v)$.

Proof. It is straightforward to see that the functions of types $(i)-(v)$ are associative polynomial functions.

Now, by Proposition 4 the polynomial function $p$ has the form

$$
p(\mathbf{x})=c_{0}+\sum_{i=1}^{n} c_{i} x_{i}, \quad c_{0}, \ldots, c_{n} \in R .
$$

Comparing the coefficients of $x_{1}$ in $p_{1}$ and $p_{2}$, we obtain the equation $c_{1}^{2}=c_{1}$. Similarly, we show that $c_{n}^{2}=c_{n}$. If $c_{1}=0$ or $c_{n}=0$, we conclude by Proposition 2 Thus we can assume that $c_{1}=c_{n}=1$. Comparing the coefficients of $x_{i}$ in $p_{i}$ and $p_{i-1}$ for $2 \leqslant i \leqslant n$, we obtain the equations $c_{1} c_{i}=c_{2} c_{i-1}$, or equivalently, $c_{i}=c_{2}^{i-1}$ and $c_{2}^{n-1}=1$. Finally, since the constant term in $p_{i}$ is $c_{0}+c_{i} c_{0}$, we must have $c_{0}=0$ unless $c_{1}=\cdots=c_{n}$.

\footnotetext{
${ }^{1}$ In fact, $m=\operatorname{mex}_{[n]}\left(J_{0}\right)$, where 'mex' stands for the minimal excluded number, well known in combinatorial game theory.
} 
Lemma 6. Let $p: R^{n} \rightarrow R$ be an associative polynomial function of the form (3). If $c_{[n]} \neq 0$, then $p$ is a symmetric function.

Proof. Let us first prove that $c_{J}=c_{J^{\prime}}$ for every $J, J^{\prime} \in[n]$ such that $|J|=\left|J^{\prime}\right|=n-1$. Setting $S=[2 n-1] \backslash\{n\}$, we see by Lemma 3 that $r_{S}^{i}=c_{[n]} c_{[n] \backslash\{n-i+1\}}$ for $i \in[n]$ and we conclude by associativity.

We now proceed by induction. Suppose that $c_{J}=c_{J^{\prime}}$ for every $J, J^{\prime} \in[n]$ such that $|J|=\left|J^{\prime}\right| \geqslant k$ for some $2 \leqslant k \leqslant n-1$ and set $c_{|J|}=c_{J}$ for every $J \subseteq[n]$ such that $|J| \geqslant k$. Fix $J_{0}$ such that $\left|J_{0}\right|=k-1$ and set $S=J_{0} \cup C_{1}$ and $m=\min \left([n] \backslash J_{0}\right) \leqslant n-1$. By Lemma 3 and associativity we have $c_{[n]} c_{J_{0}}=r_{S}^{1}=r_{S}^{m+1}=c_{n-1} c_{\left|J_{0}\right|+1}$.

The interest of Lemma 6 is shown by the following obvious result.

Lemma 7. A symmetric function $f: R^{n} \rightarrow R$ is associative if and only if the associated functions $f_{1}, \ldots, f_{n}$ satisfy the condition $f_{1}=f_{2}$.

Recall that the $n$-variable elementary symmetric polynomial functions of degree $k \leqslant n$ are defined by

$$
P_{k}(\mathbf{x})=\sum_{K \subseteq[n],|K|=k} \prod_{i \in K} x_{i} .
$$

Proposition 8. A polynomial function $p: R^{n} \rightarrow R$ such that $\operatorname{deg}(p)>1$ is associative if and only if it is of the form

$$
p(\mathbf{x})=\sum_{k=0}^{n} c_{k} P_{k}(\mathbf{x})
$$

where the coefficients $c_{k} \in R$ satisfy the conditions

$$
c_{j+1} c_{k}+c_{j} \delta_{k, 0}=c_{j} c_{k+1}, \quad j \in[n-1], k \in[n]-1 .
$$

Proof. By Proposition 5 and Lemma 6, any associative polynomial function $p: R^{n} \rightarrow$ $R$ such that $\operatorname{deg}(p)>1$ is of the form (5). By Lemma 7 such a polynomial function is associative if and only if $p_{1}=p_{2}$, that is, with the notation of Lemma 3, $r_{S}^{1}=r_{S}^{2}$ for every $S \subseteq[2 n-1]$.

Set $j=\left|S \cap C_{1}\right|, k=\left|S \cap B_{1}\right|, j^{\prime}=\left|S \cap A_{2}\right|+\left|S \cap C_{2}\right|$, and $k^{\prime}=\left|S \cap B_{2}\right|$. We have either $j^{\prime}=j-1$ and $k^{\prime}=k+1$, or $j^{\prime}=j+1$ and $k^{\prime}=k-1$, or $j^{\prime}=j$ and $k^{\prime}=k$. Therefore we get the equations

$$
\begin{array}{ll}
c_{j+1} c_{k}+c_{j} \delta_{k, 0}=c_{j} c_{k+1}, & j \in[n-1], k \in[n]-1, \\
c_{j+2} c_{k-1}+c_{j+1} \delta_{k-1,0}=c_{j+1} c_{k}, & j \in[n-1]-1, k \in[n] .
\end{array}
$$

We conclude by observing that both sets of conditions are equivalent.

Let us now consider the special case where $R$ is a field.

Proposition 9. Assume that $R$ is a field. The associative polynomial functions from $R^{n}$ to $R$ of degree $>1$ are of the form

$$
p_{a, b}(\mathbf{x})=-b+a \prod_{i=1}^{n}\left(x_{i}+b\right),
$$

where $a \in R \backslash\{0\}$ and $b \in R$.

Remark 1. The functions $p_{a, b}$ defined in (7) can be written in several equivalent forms. It is easy to see that they are associative since so are $p_{a, 0}$ and $p_{a, b}=$ $\varphi \circ p_{a, 0} \circ\left(\varphi^{-1}, \ldots, \varphi^{-1}\right)$ where $\varphi(x)=x-b$. 
Proof. Since the coefficient $c_{n}$ in (5) is nonzero by Proposition 5, we can set $a=c_{n}$ and $b=c_{n-1} / a$. Using equation (6) for $j=n-1$ and $k \geqslant 1$, we obtain $c_{k}=b c_{k+1}$, that is, $c_{k}=a b^{n-k}$. Using again equation (6) for $j=n-1$ and $k=0$, we obtain $c_{0}=-b+a b^{n}$. We conclude by observing that the function $p_{a, b}$ is associative (see remark above).

We see from the proof of Proposition 9 that the system of equations (6) has a unique solution in $\operatorname{Frac}(R)$. Therefore we can characterize the associative polynomial functions of degree $>1$ as the restrictions to $R$ of nonzero multiples of the product function, up to an affine transformation in $\operatorname{Frac}(R)$.

Proposition 10. Any associative polynomial function $p: R^{n} \rightarrow R$ such that $\operatorname{deg}(p)>$ 1 is of type (vi).

\section{FURTHER PROPERTIES}

We now investigate a few properties of the semigroup structures that we have determined.

3.1. $n$-ary groups. After classifying the ternary semigroups defined by polynomial functions, Głazek and Gleichgewicht [7] determined the corresponding ternary groups. Using the Main Theorem, we can also derive a description of the $n$ ary groups defined by polynomial functions. Recall that an $n$-ary quasigroup is given by a nonempty set $G$ and an $n$-ary operation $f: G^{n} \rightarrow G$ such that for every $a_{1}, \ldots, a_{n}, b \in G$ and every $i \in[n]$ the equation

$$
f\left(a_{1}, \ldots, a_{i-1}, z, a_{i+1}, \ldots, a_{n}\right)=b,
$$

has a unique solution in $G$. An $n$-ary group is then an $n$-ary quasigroup $(G, f)$ that is also an $n$-ary semigroup. Recall also that in an $n$-ary group, with any element $x$ is associated the element $\bar{x}$, called skew to $x$, defined by the equation $f(x, \ldots, x, \bar{x})=x$.

Proposition 11. The $n$-ary groups $(R, p)$ defined by polynomial functions $p: R^{n} \rightarrow$ $R$ of degree $\leqslant 1$ are of type $(i v)$ with $\bar{x}=(2-n) x-c$ and type $(v)$ with $\bar{x}=x$.

Proof. We immediately see that the polynomials of types $(i)-($ iii $)$ do not define $n$-ary groups. It is well known that the polynomials of types $(i v)$ and $(v)$ define $n$-ary groups 2

In general, the $n$-ary semigroups $(R, p)$ defined by type $(v i)$ are not $n$-ary groups. In the special case where $R$ is a field, we have the following immediate result.

Proposition 12. If $R$ is a field, the $n$-ary semigroup $\left(R \backslash\{-b\}, p_{a, b}\right)$, where $p_{a, b}$ is defined in (7), is an n-ary group. It is isomorphic to $\left(R \backslash\{0\}, p_{a, 0}\right)$.

3.2. Medial $n$-ary semigroup structures. We observe that all the $n$-ary semigroup structures given in the Main Theorem are medial. This is a general fact for functions of degree $\leqslant 1$ on a commutative ring. This is also immediate for the function $p_{a, b}$ defined in (7) because it is the restriction to $R$ of an $n$-ary operation that is isomorphic to a nonzero multiple of the $n$-ary product operation on $\operatorname{Frac}(R)$. From this observation it follows that, for the $n$-ary groups given in Proposition 11 the map $x \mapsto \bar{x}$ is an endomorphism.

\footnotetext{
${ }^{2}$ Polynomial functions of type $(v)$ were already considered by Dörnte [1, p. 5] in the special case of complex numbers.
} 
3.3. (Ir)reducibility of $n$-ary semigroup structures. Recall that if $(G, \circ)$ is a semigroup, then there is an obvious way to define an $n$-ary semigroup by $f\left(x_{1}, \ldots, x_{n}\right)=$ $x_{1} \circ \cdots \circ x_{n}$. In this case, the $n$-ary semigroup $(G, f)$ is said to be derived from $(G, \circ)$ or reducible to $(G, \circ)$, otherwise it is said to be irreducible. It is clear that the $n$-ary semigroups defined in types $(i)-($ iii $)$ are derived from the corresponding semigroups. However, the $n$-ary semigroups defined in type $(v)$ are not reducible. Indeed, otherwise we would have $y=y \circ 0 \circ \cdots \circ 0$ for all $y \in R$, and therefore

$$
x \circ y=x \circ(y \circ 0 \circ \cdots \circ 0)=x \circ(y \circ 0) \circ 0 \circ \cdots \circ 0=x+\omega(y \circ 0) \text {, }
$$

for $x, y \in R$, which leads to $x \circ y=x+\omega y+c$, where $c=\omega^{2}(0 \circ 0)$. We know from the Main Theorem that this function does not define a semigroup. We can prove similarly that the $n$-ary semigroups defined in type $(i v)$ are reducible if and only if $c=(n-1) c_{0}$ for $c_{0} \in R$ and, when $R$ is a field, that the semigroup $\left(R \backslash\{0\}, p_{a, 0}\right)$ is derived from a semigroup if and only if $a=a_{0}^{n-1}$ for $a_{0} \in R$.

\section{ACKNOWLEDGMENTS}

This research is supported by the internal research project F1R-MTH-PUL09MRDO of the University of Luxembourg.

\section{REFERENCES}

[1] W. Dörnte. Untersuchengen über einen verallgemeinerten Gruppenbegriff. Math. Z., 29 (1928) $1-19$.

[2] W. A. Dudek. Varieties of polyadic groups. Filomat, 9 (1995) 657-674.

[3] W. A. Dudek. On some old and new problems in n-ary groups. Quasigroups and Related Systems, 8 (2001) 15-36.

[4] W. A. Dudek, K. Glazek, and B. Gleichgewicht. A note on the axiom of $n$-groups. Coll. Math. Soc. J. Bolyai, 29 "Universal Algebra", Esztergom (Hungary), 1977, 195-202.

[5] W. A. Dudek and K. Głazek. Around the Hosszú-Gluskin theorem for n-ary groups. Discrete Mathematics, 308 (2008) 4861-4876.

[6] J. Timm. Zur gruppentheoretischen Beschreibung n-stelliger Strukturen. (German) Publ. Math. Debrecen, 17 (1970) 183-192 (1971).

[7] K. Głazek and B. Gleichgewicht. On 3-semigroups and 3-groups polynomial-derived from integral domains. Semigroup Forum, 32 (1) (1985) 61-70.

[8] M. Hosszú. On the explicit form of $n$-group operations. Publ. Math. Debrecen 10 (1963) 88-92.

[9] J. D. Monk and F. M. Sioson. On the general theory of $m$-groups. Fund. Math., 72 (1971) 233-244.

[10] E. L. Post. Polyadic groups, Trans. Amer. Math. Soc. 48 (1940) 208-350.

[11] D. Zupnik. Polyadic semigroups, Publ. Math. Debrecen 14 (1967) 273-279.

Mathematics Research Unit, FStC, University of Luxembourg, 6, Rue CoudenhoveKAlergi, L-1359 LuXembourg, LuXembourG

E-mail address: jean-luc.marichal [at] uni.lu

Mathematics Research Unit, FStC, University of Luxembourg, 6, Rue CoudenhoveKalergi, L-1359 Luxembourg, Luxembourg

E-mail address: pierre.mathonet [at] uni.lu 